\title{
Cuestiones sobre gobernanza global de los mares: un estado de la cuestión
}

Nastassja Rojas Silva*

\section{Introducción}

Abordar la gobernanza como un concepto universalmente aceptado es partir de una premisa equivocada. La definición de esta categoría va a estar atada a la aplicación que se le pretenda dar, particularmente cuando se diferencia en su aplicación en dos dimensiones: la doméstica y la internacional, escenarios en los cuales los actores o participantes de este proceso tienen diferentes posiciones de poder y organización. Es decir, se hace referencia a dos comunidades que distan la una de la otra, pese a que la gobernanza apunta a una organización horizontal de todos los que intervienen y se ven afectados por la política, el sistema internacional por su propia naturaleza anárquica y por la forma como se encuentra distribuido el poder tiene unas lógicas de comprensión muy diferentes a la esfera doméstica.

De acuerdo con el Informe sobre desarrollo mundial 2017 del Banco Mundial, la gobernanza tiene una relación intrínseca con la idea de buen gobierno, en el cual el rol del Estado va a tener cambios significativos en cuanto al desarrollo, ya que asume una posición coordinada con los demás actores sociales, lo que va a generar políticas más efectivas y estables en el tiempo, pues se adaptan a los cambios y esto solo es posible cuando las personas deciden de manera conjunta sobre el bien común. En este

\footnotetext{
* Ph.D. (c) en Derecho de la Universidad Nacional de Colombia; magíster en Relaciones Internacionales y Estudios de pregrado en Ciencias Políticas y Derecho. Profesora de tiempo completo del Departamento de Relaciones Internacionales de la Pontificia Universidad Javeriana; miembro del Grupo de Investigación en Relaciones Internacionales, América Latina e Integración, de la Pontificia Universidad Javeriana. Correo electrónico: nastassjarojas@gmail.com
} 
orden de ideas, el Banco Mundial, tomando como base a Dahl y Lukes, ha definido "gobernanza" como:

El proceso a través del cual actores estatales y no estatales interactúan para diseñar e implementar políticas dentro de un conjunto dado de reglas formales e informales que conforman y están moldeadas por el poder. Este Informe define el poder como la capacidad de grupos e individuos para hacer que otros actúen en interés de esos grupos e individuos y para lograr resultados específicos. (Banco Mundial, 2017, p. 41)

Dependiendo del contexto, los actores pueden establecer un Gobierno como un conjunto de instituciones estatales formales (organizaciones y reglas) que apliquen las políticas. También dependiendo del contexto, los actores estatales desempeñarán un papel más o menos importante con respecto a los actores no estatales, como las organizaciones de la sociedad civil y los grupos de presión empresariales. Además, la gobernanza se lleva a cabo a diferentes niveles, desde organismos internacionales hasta instituciones estatales nacionales, agencias gubernamentales locales, asociaciones comunitarias y empresariales. Estas dimensiones a menudo se superponen, lo que crea una red compleja de actores e intereses.

Cuando se traslada este concepto a la dimensión internacional, para su aplicación en un sistema con condiciones diferentes a los niveles de participación doméstica, como se señaló anteriormente, se deben abordar dos aspectos principales. Primero, las múltiples dificultades de los Estados para gestionar individualmente los problemas que se dan en su esfera doméstica, así como los llamados problemas transnacionales; esto, debido a las influencias del mundo exterior y la perforación de la soberanía estatal, producto de la globalización. Un ejemplo de ello ha sido el reconocimiento, por parte de Colombia, de apoyo y trabajo coordinado con los demás países de la región o de organizaciones multilaterales, en su lucha contra el narcotráfico. No solo es búsqueda de solidaridad, sino también de acciones concretas para enfrentar un problema que va más allá de sus propias fronteras. Para Eberwein (2010), esto podría significar que los Estados ceden una parte de su soberanía para transferirla a organizaciones internacionales o regionales en las cuales, en muchos casos, estos problemas se van a asumir como una causa supranacional.

El segundo aspecto es la participación de actores diferentes al Estado, como lo son las Organizaciones No Gubernamentales (ONG), partiendo de un problema de legitimidad y por ende de actuación en el sistema internacional. En el caso de los Estados, la legitimidad se desprende del derecho internacional (Wolf-Dieter, 2013, p. 4), pero partiendo de la idea de que la comunidad internacional se encuentra lejos de ser una sociedad democrática, especialmente cuando se refiere a la influencia de otros 
actores, pues las discusiones se van a dar en torno a la necesidad y funcionalidad de su participación, siendo aceptada finalmente su existencia por parte de los Estados de manera forzada, al no poder desconocer el impacto que generan en sus sociedad e incluso en muchos de los procesos de tomas de decisiones; de esta manera, llegan a ser avaladas como un actor del sistema internacional por la Organización de las Naciones Unidas (ONU). ${ }^{1}$

Probablemente, el reconocimiento de estos actores también se deba en alguna medida a los mismos impactos de la globalización, al ser muchas de ellas de carácter transnacional; el trabajo en red por parte de muchas de estas organizaciones les ha asegurado un asiento en las relaciones internacionales. Los procesos de globalización producirán también efectos importantes sobre los Estados y su posición en el sistema internacional, pero esto no debe entenderse como el desplazamiento de estos o su obsolescencia. Esto va a significar para las unidades nacionales la participación de otros actores también importantes y reconocidos globalmente, sin que esto implique la creación de un Gobierno mundial.

De acuerdo con la ONU, las aguas oceánicas abarcan un área inmensa de la superficie terrestre, y de esta manera constituyen una fuente importante de alimentación y transporte, lo cual no representó una mayor preocupación para su regulación, más allá de temas de seguridad continental para los Estados. Sin embargo, con los avances tecnológicos, los Estados y actores privados internacionales empezaron a sacar mayor provecho de los espacios marítimos e incluso de aquellos espacios que se creían imposibles de explorar, como lo fue la Alta Mar. Con este desarrollo, en cuanto a la capacidad de navegación y particularmente de explotación, se evidencia la necesidad de generar nuevas formas de gestión de los espacios marítimos, sin ser suficiente la sola delimitación del ejercicio de la soberanía de los Estados, sino la gestión coordinada de problemas que trascienden las fronteras y que por lo tanto necesitan de una administración conjunta y coordinada. Algunos de los principales problemas que serán objeto del trabajo internacional son:

- La libertad de navegación y uso de los mares.

- Asuntos de protección del medio marino y la biodiversidad.

- Condiciones para el transporte marítimo y la contaminación.

- Gestión de aguas internacionales (Alta Mar) y de las aguas polares.

- Delitos relacionados con los espacios marítimos, especialmente la piratería.

1 De acuerdo con la Carta de la onu en su Artículo 71, las Organizaciones No Gubernamentales pueden colaborar con esta como entidades consultivas en el Consejo Económico y Social Colaborar con el sistema de la onU y posteriormente con la creación del Departamento de Información Pública pueden acreditarse y asociarse ante la sección de las Organizaciones no gubernamentales de este Departamento. 
No obstante, pese a los avances que se van a presentar en la administración de los mares, se podría afirmar que plantear una división de los espacios marítimos y determinar el ejercicio de la soberanía estatal sobre estos no ha sido suficiente para una gestión efectiva de los espacios marítimos, lo cual impide llegar a un sistema real de gobernanza global, particularmente cuando se hace referencia a las aguas internacionales, objeto de grandes debates en cuanto a su uso por parte de los Estados.

En este orden de ideas, este capítulo está orientado a presentar el estado actual de la gobernanza global marítima y la importancia que representa gestionar de manera coordinada la Alta Mar. Para ello, el texto presentará primero una conceptualización de la gobernanza en su dimensión internacional; segundo, el papel del derecho internacional público en la gobernanza global, y tercero, la situación en cuanto a la gobernanza de las aguas internacionales.

\section{La gobernanza en el escenario internacional: conceptos y elementos orientadores}

Lo primero que se debe aclarar antes de llegar al concepto de gobernanza en este escenario es que no se hace referencia a la idea de una gobernanza internacional, sino de una gobernanza global. La comprensión de internacional estaría haciendo referencia a que si bien las instituciones que orientarían las acciones y las políticas que allí se generen estarían presentes en la mayor parte de los Estados, son gestionadas de manera independiente entre ellas, siendo al final solo un conjunto de normas estatales. Lo global hace referencia a aquellas políticas y acciones que se gestionan de manera conjunta y coordinada por los Estados y otros actores no tradicionales; sin embargo, no debe confundirse con la idea de implementar un Gobierno mundial. La naturaleza anárquica del sistema sigue estando presente, solo que se van a generar acuerdos para dar un mejor tratamiento a los problemas transnacionales. Estos problemas o retos internacionales contemporáneos, en palabras de Inge y Blondin (2015), necesitan de una gestión global para poder garantizar la defensa de lo que se conoce como bienes públicos globales. ${ }^{2}$ Para estos autores, la mayoría de los problemas de esta escala

2 Los bienes públicos globales son aquellos que por su propia naturaleza su producción requieren un esfuerzo conjunto de todos los Estados, por cuanto de ellos se favorece toda la comunidad internacional, por más generaciones que la actual, siendo internacional, interpersonal e intergeneracional. Con el paso del tiempo y el afianzamiento de este concepto, se han incluido diferentes categorías sobre estos bienes, extendiéndolos a bienes naturales y culturales, como por ejemplo aquellos que proporcionan una utilidad directa, cuya provisión permite obtener beneficios directos que incrementan la utilidad de agentes económicos; en esta categoría se podrían ubicar los océanos. 
necesitan la adopción de medidas internacionales, e incluso llegan a considerar que en muchos casos la estrategia adecuada es acordar modelos de soberanía compartida.

Ante la necesidad de intervenir de manera coordinada, los Estado optan por configurar una buena parte de sus relaciones por medio de organizaciones o subsistemas que funcionan como una suerte de tramitadores de los diferentes intereses de quienes participan, y que por medio de procesos y reglas particulares de la organización generarán propuestas conjuntas de gestión de los problemas. Por esta razón, las organizaciones intergubernamentales juegan un rol de gran importancia en este sistema internacional, sobre todo ante el surgimiento y reconocimiento de problemas de carácter internacional que, como se mencionó, por su propia naturaleza los Estados no pueden solucionar de manera individual. Así, la creación de instituciones internacionales que los Estados, en virtud de su soberanía, deciden acatar permite resolver esos problemas que son de carácter internacional.

Como señala Charles-Philippe David (2008), la construcción del concepto de gobernanza global tiene una estrecha relación con los postulados de la escuela idealista en cuanto a su comprensión de la paz mediante el derecho, es decir, por medio de institucionales de tipo internacional, entiéndase normas y organizaciones, ya que su existencia y reconocimiento por parte de los Estados reducirían las posibilidades del uso de la fuerza como una forma de "resolver" los conflictos. Este punto de confluencia entre la visión de construcción de una paz internacional y la efectividad real del derecho internacional está sostenida sobre la idea de la configuración de la gobernanza global, al considerar la existencia de un orden internacional bajo del funcionamiento de normas que trascienden a los Estados (creadas por ellos mismos), resguardadas principalmente por organizaciones internacionales con la participación de actores no tradicionales como la sociedad civil. A su vez, esta misma gobernanza, en palabras de David Held (1995), debe procurar el restablecimiento de la asimetría entre los generadores de decisiones en el sistema internacional. Es decir, la gobernanza global nace y sostiene al mismo tiempo el equilibrio del propio sistema internacional, así como la efectividad de las instituciones internacionales se basa en el concepto de la gobernanza global. A continuación, se exponen diferentes conceptos de gobernanza desarrollados en diferentes textos que han aportado a la construcción teórica.

De acuerdo con la Real Academia Española, la gobernanza se entiende como el arte o manera de gobernar que se propone como objetivo el logro de un desarrollo económico, social e institucional duradero, promoviendo un sano equilibrio entre el Estado, la sociedad civil y el mercado de la economía (Real Academia Española, 2014). 
Por su parte, Mayntz (2001) expresa que la gobernanza se entiende como:

La realización de relaciones políticas entre diversos actores involucrados en el proceso de decidir, ejecutar y evaluar decisiones sobre asuntos de interés público, proceso que puede ser caracterizado como un juego de poder, en el cual competencia y cooperación coexisten como reglas posibles; y que incluye instituciones tanto formales como informales. La forma e interacción entre los diversos actores refleja la calidad del sistema y afecta a cada uno de sus componentes; así como al sistema como totalidad. (p. 222)

En cuanto a su elevación al plano internacional, en el texto Los Estados nacionales y la gobernanza global (2002), la misma autora establece que:

Las organizaciones internacionales, gubernamentales y no gubernamentales, componen conjuntamente lo que por lo general se denomina gobernanza global. La gobernanza, por definición, se refiere a la solución de problemas colectivos; no se refiere a la dominación de por sí. Las organizaciones internacionales, tanto gubernamentales como no gubernamentales, están comprometidas en procesos para la solución de problemas colectivos, y los problemas de los cuales deben ocuparse aparecen enumerados en sus estatutos y su misión establecida. Esto es cierto en instituciones supranacionales, tales como las Naciones Unidas; organizaciones gubernamentales internacionales como, por ejemplo, la Organización Internacional del Trabajo, el Banco Mundial y la Organización Mundial del Comercio. (p. 30)

En este orden de ideas, siguiendo el recorrido conceptual expuesto anteriormente y las características que se derivan de la ubicación de esta comprensión en el escenario internacional, se presentan los atributos de la gobernanza global:

Tabla 1. Atributos de la gobernanza global

\begin{tabular}{|c|l|}
\hline Atributo & \multicolumn{1}{c|}{ Contenido } \\
\hline 1. Dimensiones & $\begin{array}{l}\text { Se pueden identificar dos dimensiones en cuanto a gobernanza: una nacional o } \\
\text { doméstica y una internacional o global. Si bien el concepto tiene una asociación } \\
\text { conceptual con la gobernanza doméstica, no debe usarse de manera análoga en } \\
\text { la dimensión internacional, debido a las diferencias notables de ambas esferas } \\
\text { en cuanto a la organización social e institucional. Por esta razón, tampoco } \\
\text { deben entenderse como sinónimos gobernanza global y gobierno mundial, } \\
\text { por cuanto aquí no se está hablando de autoridades reconocidas como entes } \\
\text { supranacionales. }\end{array}$ \\
\hline
\end{tabular}




\begin{tabular}{|c|c|}
\hline Atributo & Contenido \\
\hline 2. Rol del Estado & $\begin{array}{l}\text { El Estado sigue siendo el actor protagónico en la gobernanza global, pero } \\
\text { con frecuencia tiene que negociar procesos y decisiones con otros actores del } \\
\text { sistema internacional. Por este motivo, los mecanismos intergubernamentales } \\
\text { como coordinadores de la acción colectiva seguirán siendo importantes, incluso } \\
\text { como canalizadores del diálogo entre los Estados y los actores no tradicionales; } \\
\text { sin embargo, estos entes intergubernamentales no existirían sin el Estado. }\end{array}$ \\
\hline $\begin{array}{l}\text { 3. Impacto sobre } \\
\text { la soberanía }\end{array}$ & $\begin{array}{l}\text { El concepto que se plantea en este texto no considera que la construcción y el } \\
\text { fortalecimiento de la gobernanza global atente contra la existencia del Estado } \\
\text { en el sistema internacional al afectar la libertad de decisión y acción de los } \\
\text { estos; por el contrario, representa un proceso de transformación que aseguraría } \\
\text { su supervivencia en el sistema, ya que les permite gestionar de manera más } \\
\text { efectiva los problemas transnacionales. }\end{array}$ \\
\hline $\begin{array}{l}\text { 4. Participación } \\
\text { de actores no } \\
\text { tradicionales }\end{array}$ & $\begin{array}{l}\text { Se reconoce la participación de las organizaciones no gubernamentales, } \\
\text { partiendo de las dificultades que estas van a tener para ser reconocidas como } \\
\text { sujetos en el sistema internacional y que son las mismas organizaciones inter- } \\
\text { gubernamentales, como por ejemplo la onu, que reconocerán su participación } \\
\text { e importancia. No obstante, no se puede negar que su impacto en las decisiones } \\
\text { en muchas ocasiones estará supeditado a la voluntad de los Estados. }\end{array}$ \\
\hline $\begin{array}{l}\text { 5. Instituciones } \\
\text { interguberna- } \\
\text { mentales }\end{array}$ & $\begin{array}{l}\text { Debido a la naturaleza particular del sistema internacional, es necesaria la } \\
\text { existencia de instituciones de gobernanza global; por esta razón, es fundamen- } \\
\text { tal el estudio de las organizaciones y normas internacionales desde su mismo } \\
\text { proceso de creación, ya que, si bien es de gran importancia el esfuerzo de } \\
\text { establecer normas para limitar el ejercicio del Estado frente a sus pares y frente } \\
\text { a sus mismos ciudadanos, para hablar de gobernanza global es necesario que } \\
\text { todos los Estados, sin importar su posición en el sistema (entiéndase Estados } \\
\text { periféricos, potencias regionales, potencias mundiales) participen de manera } \\
\text { activa e independiente. }\end{array}$ \\
\hline $\begin{array}{l}\text { 6. Obstáculos en } \\
\text { su desarrollo }\end{array}$ & $\begin{array}{l}\text { De la característica anterior se derivan dos problemas fundamentales que obsta- } \\
\text { culiza el desarrollo de la gobernanza a nivel global. Primero, no se puede negar } \\
\text { que el desarrollo de las normas y organizaciones, así como la participación de } \\
\text { actores no tradicionales está supeditado a la voluntad política de los Estados. } \\
\text { Pese a que la soberanía empezará a ser entendida como una cualidad relativa } \\
\text { y que la actuación de los Estados tiene límites, con frecuencia ignoran la exis- } \\
\text { tencia de las instituciones que intentan delinear su comportamiento. Segundo, } \\
\text { de acuerdo con la Resolución } 2625 \text { de la Asamblea General de las Naciones } \\
\text { Unidas, los Estados son parte de un principio de igualdad soberana (esto se } \\
\text { entiende como que son iguales derechos y deberes, más allá de las diferencias } \\
\text { socioeconómicas y políticas). Sin embargo, la realidad del sistema internacio- } \\
\text { nal es que las diferencias entre los Estados constituyen un determinante del } \\
\text { impacto de su participación, la cual en gran medida está condicionada por } \\
\text { sus capacidades duras (PIB, población, territorio, gasto militar y presencia } \\
\text { económica) y por sus capacidades blandas (PIB per cápita, índice de desarrollo } \\
\text { humano, paridad del poder adquisitivo). Esto afirma que sí existe una jerarqui- } \\
\text { zación entre los Estados. }\end{array}$ \\
\hline
\end{tabular}




\section{El derecho internacional público como elemento coordinador de asuntos marítimos}

Es frecuente que cuando se hace alusión a las instituciones jurídicas como un elemento esencial en el equilibrio internacional, se pone en tela de juicio el propio sentido y la naturaleza jurídica del derecho internacional público y su existencia, ya que es comparado con toda la estructura y las características del derecho doméstico, principalmente cuando se llama la atención en cuanto a la no identificación de un órgano legislativo superior creador de las normas, y además la inexistencia de un sistema jurisdiccional. De esta manera, algunos juristas sostendrán que en la ausencia de un órgano generador de normas y otro que imparta sanciones no se puedo hablar en sentido estricto de un sistema jurídico.

Por esta razón, algunos autores van a considerar que el derecho internacional, en virtud de tener su origen en los mismos Estados, otorga a estos la posibilidad de decisión sobre las formas, la intensidad y jerarquía en cuanto a la incorporación de las instituciones internacionales. En otras palabras, cada Estado cuenta con la autonomía de asimilar la concepción que de su mismo ordenamiento se derive. Esto nos lleva a que la idea de una gobernanza global, realmente efectiva y de verdaderas dimensiones globales va a depender de la voluntad política de los Estados, ya que la idea de lo global, como se dijo al inicio, consiste en que se generen las instituciones en un núcleo central y que se expanda más allá de las fronteras estatales; no es suficiente con que estas normas se adapten en el interior de cada Estado. No obstante, siguen vigentes sus ánimos de coordinación de las relaciones pacíficas entre los actores internacionales. Pero, al menos hasta los avances actuales en cuanto a la consolidación del derecho internacional público (DIP), pese a esta pretensión de coordinación y de asegurar la misma existencia del Estado, se evidencia que la condición principal para su aplicación es el consentimiento de los Estados, lo cual genera discusiones importantes sobre la obligatoriedad de los instrumentos emanados de las instituciones internacionales. ${ }^{3}$

Sin embargo, pese a los detractores, en el ámbito más amplio de la comunidad internacional, las normas que regulan problemas como la guerra, los sistemas de armas, los crímenes de guerra, los derechos humanos y el medio ambiente, entre otras, han transformado y delimitado el orden estatal, incorporando en las políticas nacionales nuevas formas y niveles de responsabilidad y de gobernanza. En consecuencia, las fronteras entre las sociedades nacionales han dejado de tener un único significado

3 La obligatoriedad de los tratados no nace del principio de legalidad, la base para su cumplimiento se encuentra en el principio "Pacta sunt servanda" (todo tratado en vigor obliga a las partes y debe ser cumplido por ellas de buena fe). 
jurídico y moral que una vez tuvieron en la época de la soberanía tradicional-clásica (Held, 2010).

La soberanía del Estado no debe ser entendida como un principio devastador del derecho internacional, sino como el punto de inicio en la comprensión de este sistema jurídico particular. Esta cualidad representa la relevancia en la voluntad y el consentimiento del Estado en el proceso de creación, desarrollo progresivo y aplicación del derecho internacional, más aún cuando la soberanía del Estado ha pasado de ser una condición absoluta a ser relativa. A pesar de las discusiones que se puedan dar frente a la existencia de norma internacionales, su importancia en el sistema es innegable.

Es necesario entonces, para construir un sistema efectivo de gobernanza global, superar la discusión de la incorporación como una condición de la funcionalidad del DIP en contraposición a la soberanía estatal. Las mismas instituciones internacionales, en acuerdo con los sujetos que de ellas participan, están llamadas a abandonar las concepciones monistas y dualistas de la relación entre los dos sistemas jurídicos, ya que esto seguirá siendo una condicionante para el desarrollo de la sociedad internacional y de la convivencia pacífica entre Estados. Por tal razón, es de vital importancia superar esta discusión doctrinal y proponer de manera coordinada un sistema de acoplamiento.

De allí viene la importancia de las normas internacionales para regular los asuntos marítimos, al considerar que el inicio para regular y controlar los objetivos de expansión de los Estados sobre los espacios marítimos fue la adopción de las Convenciones de Ginebra de 1958 (su importancia viene dada al determinar lo relacionado con el mar territorial, aguas internacionales, la pesca y la conservación de los recursos en estos espacios), así como la Convención de las Naciones Unidas sobre el Derecho del Mar de 1982. Esta última representa un importante aporte al reunir en un solo texto lo establecido sobre el mar hasta el momento y el derecho del mar consuetudinario (Couvreur, 2007).

\section{Organizaciones intergubernamentales}

Cuando se hace referencia a la gobernanza en su dimensión global, las organizaciones intergubernamentales son un actor protagónico, ya que recae en ellas la función de coordinación del sistema internacional, por cuanto se encargan de velar por esa estructura que se deriva de estas; pero no se deben confundir, de ninguna manera, con la creación de un Gobierno supranacional. Su importancia deviene al ser el escenario donde los Estados encuentran la posibilidad de cooperar para la solución de 
problemas que trascienden sus fronteras y de que, de manera aislada, serían imposibles de resolver.

El orden mundial producto de la Segunda Guerra Mundial se va a caracterizar por la multiplicación de instituciones internacionales y regionales, de las cuales se van a derivar un sin número de convenciones y acuerdos de tipo político y económico. El ejemplo más claro sobre esta institucionalización de las relaciones fue la creación y el desarrollo de la ONU. Este nuevo orden va a generar naturalmente una serie de nuevas responsabilidades entre los Estados y de estos con sus ciudadanos, hecho que ubicará a los individuos como un sujeto en el sistema internacional, y que en caso de ser violados estos acuerdos existirán cortes con jurisdicción internacional ${ }^{4}$ y regional ${ }^{5}$.

Sin embargo, estas organizaciones van a encontrar una gran dificultad en cuanto al alcance y la obligatoriedad de sus decisiones. Si bien los Estados en virtud de su soberanía deciden someterse a las directrices creadas por ellos mismos, pero de manera colectiva, son muchos los casos en los cuales las organizaciones intergubernamentales son señaladas de inoperantes, por cuanto las posibilidades de sanciones efectivas son restringidas. Lo cierto es que la capacidad de estas instituciones sobre los actores del sistema internacional dependerá en gran medida de las capacidades materiales e inmateriales del Estado que pretenden someter a una decisión; es decir, cuando se refiere a estados periféricos o Estados con capacidades limitadas la capacidad de actuación de la organización será mayor; no obstante, pese a que los Estados con mayores capacidades con frecuencia evaden las acciones de estas instituciones, la existencia de normas e instituciones los obliga a negociar sus acciones en el plano internacional.

El Tribunal Internacional del Derecho del Mar es un órgano judicial independiente creado por mandato de la Convención de las Naciones Unidas sobre el Derecho del Mar, con el propósito principal de aplicar las normas establecidas en ese instrumento, tales como delimitación de zonas marítimas, navegación, protección y preservación del medio marino, entre otras. Este tribunal se encuentra abierto a los Estados que hacen parte de la Convención, así como a las empresas y entidades privadas sobre asuntos relacionados con explotación de los fondos marinos.

4 La Corte Internacional de Justicia, siendo el principal órgano judicial de la onU, encargado de dirimir las controversias de orden jurídico entre Estados conforme al Derecho Internacional.

5 La Corte Interamericana es un tribunal regional de derechos humanos, siendo la responsable de interpretar y aplicar la Convención Americana. 


\section{Gestión y gobernanza global de los espacios marítimos}

De acuerdo con la Política Marítima Integrada planteada por la Comisión Europea ${ }^{6}$ el $60 \%$ de los océanos se encuentra por fuera de los límenes de jurisdicción nacional, lo cual se traduce en que se constituyen como recursos compartidos y aun cuando se encuentre bajo el ejercicio soberano de un Estado determinado existirán ciertas restricciones para este, en virtud de los derechos de terceros Estados. Ante esta necesidad del uso de los océanos y los mares por ser fuente de alimento, empleo, comercio y recreo, se fue generando al mismo tiempo la necesidad de gestionar marcos institucionales y legales con la partición conjunta y coordinada de diferentes Estados y actores para lograr estrategias efectivas y perdurables en el tiempo en la gestión de estos espacios.

De acuerdo con Edmundo Vargas (1975), los Estados con mayores capacidades en el sistema internacional, en los primeros momentos de gestión y organización de los mares, no se encontraban interesados en la posibilidad de proponer normas para la regulación de estos espacios, particularmente cuando se intentaba referir a la idea de limitar la libertad del mar por una cuestión de seguridad. No obstante, el proceso de creación de las instituciones que conformarían el derecho del mar se encontraba en marcha. Esta etapa tradicional o clásica del DIP, en sus inicios, va a conservar la idea de libertad de los mares, entendida como "res communis ómnium". Esto, como producto de la posición de los Estados más poderosos, es decir, aquellos que tenían un desarrollo naval importante, lo que solo era posible si se tenían los medios económicos y militares necesarios. Sin embargo, esta concepción del mar empezará a ser cuestionada por las acusaciones sobre conductas arbitrarias que ocurrían en estos espacios.

Estas serias dificultades y otros problemas sobre el uso y la navegación de los mares llevaron a gran parte de la comunidad internacional a la Conferencia de Codificación del Derecho Internacional (1930) en el marco de la Sociedad de Naciones. Posteriormente, con la creación de la ONU, en 1958, se da la primera Conferencia Internacional en Ginebra para Codificar el derecho del mar. Este proceso lleva al antecedente más importante en este proceso de codificación, la III Conferencia de Naciones Unidas, reunión que produjo la Convención de Naciones Unidas para el Derecho del Mar en 1982 (oNU, 2004). Esta institucionalización de las normas referidas a los asuntos del

6 La Unión Europea (UE) ha llevado la delantera en cuanto a la construcción de la gobernanza internacional de los océanos, representando uno de sus principales avances: la adopción de la Política Marítima Integrada, la cual tiene como propósito coordinar las políticas en sectores marítimos específicos. Para la UE, la ausencia de normas o la observancia de estas suponen un peligro para los ecosistemas marinos, por ello la solución no es solo la creación o la sustitución de las instituciones ya existente, sino una coordinación entre ellas, lo cual significa una verdadera gobernanza. 
Mar va a dar cabida a nuevas interpretaciones y pretensiones de los Estados en cuanto a su soberanía sobre las aguas.

La primera etapa del derecho del mar tradicional o clásico va a comprender aquel periodo desde la existencia de los Estados-nación, ya que el mar era entendido desde aquel entonces como fuente de transporte y de alimentación, atribuyéndose la primera codificación del derecho del mar al Imperio romano, que establecerán el concepto de Mare Nostrum para hacer referencia al mar que el imperio reclamaba como suyo (ONU, 2004). Posteriormente, para 1609, surgirá un nuevo concepto planteado por Hugo Grocio, denominado Mare Liberum, el cual establecerá principalmente que los mares no pueden limitarse a la jurisdicción de un Estado. No obstante, a este concepto se presentaría una excepción por parte de Inglaterra, el Mare Clausum, lo cual representaría futuras discusiones entre los conceptos de mares abiertos y mares cerrados, llegando por primera vez a una comprensión de división del mar, una porción con ejercicio pleno de la soberanía de los Estados y un segundo espacio de alta mar. Pero esto no fue suficiente para solventar los problemas que se venían presentando en cuanto al dominio de este espacio, lo cual permitió que esta temática siguiera estando presente en la agenda internacional. De esta forma, en 1958 tuvo lugar la Primera Conferencia de Naciones Unidas sobre Derecho del Mar, pero esta conferencia y la siguiente no lograrían mayores avances en cuanto a la gestión y soberanía sobre los mares. Solo hasta 1974, en la Tercera Conferencia, se logra avanzar sobre un acuerdo en cuanto a los fondos marinos, la plataforma continental, alta mar, la zona económica exclusiva y el mar territorial, avances que llevaron el 10 de diciembre de 1982 al inicio de las firmas de la Convención de las Naciones Unidas sobre Derecho del Mar (Convemar).

La convención se crea como el principal instrumento de coordinación entre los Estados en esta materia, pero no se impone como un Gobierno supranacional, sino como una institución de gobernanza sin Gobierno, ya que rige un principio de igualdad entre los Estados. Tampoco debe confundirse la existencia de instancias superiores para la solución de controversias con la existencia de un Gobierno mundial. Es en virtud de la soberanía de la cual gozan los Estados que al momento de ratificar la convención estos podrán elegir los medios a los que decidirían someterse en caso de controversias en cuanto a interpretación o aplicación del instrumento (el Tribunal Internacional del Derecho del Mar, la Corte Internacional de Justicia, un tribunal arbitral o un tribunal arbitral especial).

Con el tiempo, la mayoría de las discusiones van a rondar particularmente los temas de delimitación de zonas marítimas, siendo este un trabajo de carácter necesariamente internacional, porque sirve para superar un conflicto de pretensiones de dos o más Estados y tiene sentido si, una vez completada la Convemar, se impone jurídicamente a los Estados en conflicto. En este orden de ideas, se podría determinar que la convención, junto con los instrumentos que ella misma crea, genera mayor 
claridad sobre la expansión y comprensión del ejercicio de soberano de los Estados sobre el mar, siendo esto asumido por toda la comunidad internacional (Treves, 1998). No obstante, pese a lo establecido sobre el manejo de las aguas internacionales, es uno de los espacios que presenta mayores problemas en cuanto a su gestión y control, especialmente por ser una zona de difícil acceso por parte de todos los Estados, cuestión que se tratará en el siguiente apartado.

De esta manera, la Convención de las Naciones Unidas para el Derecho del mar resulta de gran importancia cuando nos referimos a la coordinación y gestión de estos espacios, por cuanto es el instrumento que establece el marco fundamental para entender y gestionar todos los asuntos relacionados con la jurisdicción, los derechos y deberes (navegación, sobrevuelo, exploración y explotación de recursos, conservación y contaminación, pesca y tráfico marítimo) de los Estados en los espacios marítimos. ${ }^{7}$ El proceso de implementación de lo establecido en la Convención, aunado a lo dispuesto por la existente Organización Marítima Internacional, da pie a la creación de diferentes órganos; entre ellos, los de mayor relevancia son:

\begin{tabular}{|l|l|}
\hline $\begin{array}{l}\text { Tribunal Internacional } \\
\text { del Derecho del Mar }\end{array}$ & $\begin{array}{l}\text { De acuerdo con el anexo VI de la Convemar, es el escenario de solución de } \\
\text { controversias sobre la interpretación o aplicación de la misma convención, } \\
\text { y es integrado por 21 miembros independientes. }\end{array}$ \\
\hline $\begin{array}{l}\text { Autoridad } \\
\text { Internacional de los } \\
\text { Fondos Marinos }\end{array}$ & $\begin{array}{l}\text { Por medio de esta los Estados que conforman el acuerdo gestionan las } \\
\text { actividades relativas a los recursos naturales en los fondos marinos fuera } \\
\text { de los límites estatales. De ahí deriva su importancia, al ser una instancia } \\
\text { de coordinación de aquellos espacios que trascienden los espacios nacio- } \\
\text { nales (artículo 156 de la Convención). }\end{array}$ \\
\hline
\end{tabular}

\section{Aguas internacionales: coordinación entre Estados y participación de actores no gubernamentales}

Como se mencionó en apartados anteriores, suponiendo que la creación y el reconocimiento de las normas e instituciones relacionadas con los asuntos marítimos resuelven buena parte de los problemas de los Estados, se van a considerar estos patrones como un paso importante en la consolidación de la gobernanza global de

$7 \quad$ Esta convención establece importantes parámetros en cuanto al uso del océano y su soberanía: la creación de derechos de libertad de navegación; establecimiento de los límites territoriales del mar a 12 millas de la costa; establecimiento de zonas económicas exclusivas a 200 millas de la costa; creación de normas para la extensión de los derechos en la plataforma continental a 350 millas de la costa; creación de una autoridad internacional de los fondos marinos. 
los mares. Una de las zonas que más despierta controversia cuando se habla de su gestión, y particularmente sobre los límites que deben observar todos los países cuando hacen uso de este espacio, es alta mar. Es preciso reconocer que en la realidad los Estados con las mayores capacidades materiales en el sistema internacional vienen ejerciendo un poderío importante en estas zonas y sirviéndose de ella para fines económicos y científicos, pero de estas se desprenden consecuencias que afectan a la comunidad internacional, como los problemas medio ambientales, sin contar con los delitos cometidos en estas aguas internacionales.

De acuerdo con la Convemar, en su artículo 86, alta mar son aquellas partes del mar no incluidas en el mar territorial, aguas interiores, aguas archipelágicas o zona económica exclusiva. Por esto, es de gran importancia que ningún Estado someta cualquier parte de la alta mar a su soberanía. Asimismo, el derecho que tienen los Estados sin litoral de acceder al mar y desde el mar ejercer los derechos que se señalan en la convención, particularmente en cuanto a la libertad de alta mar y el patrimonio común de la humanidad, deben considerar los intereses de otros Estados en su ejercicio de la libertad de la alta mar y con objetivos exclusivamente pacíficos.

Artículo 87. Libertad de la alta mar. La alta mar está abierta a todos los Estados, sean ribereños o sin litoral. La libertad de la alta mar se ejercerá en las condiciones fijadas por esta Convención y por las otras normas de derecho internacional. Comprenderá, entre otras, para los Estados ribereños y los Estados sin litoral:

a) La libertad de navegación;

b) La libertad de sobrevuelo;

c) La libertad de tender cables y tuberías submarinos, con sujeción a las disposiciones de la Parte VI;

d) Libertad de construir islas artificiales y otras instalaciones permitidas por el derecho internacional, con sujeción a las disposiciones de la Parte VI;

e) La libertad de pesca, con sujeción a las condiciones establecidas en la sección 2;

f) La libertad de investigación científica, con sujeción a las disposiciones de las Partes VI y XIII. (ONU, 1985)

Sin embargo, de los principales problemas que se van a presentar en la alta mar es que al tener importantes recursos minerales, que anteriormente no eran objeto de ser explotadores por las limitaciones tecnológicas, hoy están en la mira de muchas naciones que han generado desarrollos tecnológicos capaces de llegar a zonas de difícil explotación; además, pese a los avances que presentará la Convemar, no se va a contar con los mecanismos suficientes para garantizar una administración coordinada sobre estos espacios. 
No obstante, en algunos pocos escenarios se han intentado promover prácticas responsables en las aguas internacionales. Uno de los grandes avances que se debe destacar, en cuanto a protección y gobernanza de los océanos, es la iniciativa para la consolidación del tratado de la onU sobre la protección de la vida marina en alta mar y el uso sostenible de estas zonas ${ }^{8}$. Esta idea logra calar en el escenario institucional pese a la oposición de algunos Estados, al considerar que esto afectaría sus propios intereses económicos. Este avance no se habría logrado sin el impulso de la Alianza para el Alta Mar, ${ }^{9}$ lo que evidencia que si bien las ONG no son protagonistas en cuanto a la gobernanza global, sí logran movilizar temas de interés internacional.

En este escenario de la gestión de estas aguas internacionales, las ONG van a empezar a tener una participación especial. De acuerdo con el artículo 169 de la convención, el Secretario General, con el aval del Consejo, podrá adoptar disposiciones oportunas para la realización de consultas y la cooperación con organizaciones internacionales y con ONG reconocidas por el Consejo Económico y Social de las Naciones Unidas. Por ello, anteriormente se mencionaba la importancia que tendrá el reconocimiento de las ong por parte de la onU. No obstante, como ha sucedido en otro tipo de problemáticas, estas organizaciones han sido cuestionadas en cuanto a su legitimidad para participar como su alcance en las decisiones tomadas.

\section{Caso Arctic Sunrise}

Este fue un asunto revisado por el Tribunal Internacional del Mar, interpuesto por el Reino de los Países Bajos contra la Federación de Rusia, producto de varios hechos ocurridos a finales de 2013. Esta embarcación llamada Arctic Sunrise ${ }^{10}$ fue usada durante una protesta en una plataforma petrolera de la empresa Gazprom, protestas que no cesaron en los siguientes días y que tuvieron enfrentamientos con las autoridades

\footnotetext{
$8 \quad$ En cuanto la gobernanza de la pesca en alta mar, esta se encuentra regida por las reglas determinadas en el apartado viI: alta mar de la Convemar y en el Acuerdo sobre las Poblaciones de Peces; sin embargo, se ha evidenciado que este marco no resulta suficiente y que muchos Estados se han aprovechado del uso de esta zona.

9 La Alianza para el Alta Mar es una asociación de organizaciones que tienen el propósito de construcción de una posición y voz común para promover la conservación de a lta mar. La alianza está formada actualmente por 27 oNG, además de la Unión Internacional para la Conservación de la Naturaleza (UICN).

10 El Arctic Sunrise es una embarcación rompehielos y buque de investigación que forma parte de la flota internacional de Greenpeace, para esta protesta la tripulación estaba conformada por 30 activistas de 18 nacionalidades distintas.
} 
de Rusia, siendo finalmente remolcados y apresados los 30 ocupantes del barco y fueron acusados de vandalismo y piratería.

El caso se va a centrar principalmente en la solicitud de medidas provisionales presentada por Holanda en relación con estas detenciones, debido a que la embarcación que estaba siendo operada por Greenpeace llevaba enarbolada la bandera de los Países Bajos. De acuerdo con los argumentos de este país, en el momento de la detención, la embarcación se encontraba dentro de la zona económica exclusiva de Rusia y fue remolcado al puerto ruso de Murmansk. El tribunal ordenó que Arctic Sunrise $\mathrm{y}$ todas las personas fueran liberadas y se les permitiera abandonar el territorio ruso a cambio de una especie de fianza por parte de Holanda. Fue así como, pasados dos meses de detención, fueron puestos en libertad.

Sin embargo, Rusia fue renuente al arbitraje por parte del tribunal, al declarar que cuando ratificó la Convención del Mar se estableció que no aceptaba todo lo relacionado con el arbitraje, en particular el carácter vinculante de decisiones sobre el ejercicio de derechos soberanos. Pese al retiro de los cargos en contra de los tripulantes, el caso no concluyó ahí, debido a que Holanda había interpuesto en su petición que Rusia violó la Convención del Mar debido a que el Arctic Sunrise era de bandera holandesa (considerándose territorio holandés). Durante todo el proceso se evidenció el constante rechazo de la Federación de Rusia a la jurisdicción del tribunal y su no comparecencia, lo cual representa uno de los obstáculos más graves que enfrentan las instituciones de gobernanza global, pues necesitan contar con la voluntad del Estado. Otro de los fenómenos de particular relevancia en este caso es el papel que desempeño una ONG. Ante la posición de Rusia frente al tribunal, con el propósito de apoyar la solicitud de los Países Bajos para las medidas provisionales, Greenpeace decide presentar un amicus curiae como parte del expediente (este escrito señalaba la aplicabilidad del derecho internacional de los derechos humanos a las acciones de las autoridades de Rusia, alegando la violación de los derechos de quienes estaban a bordo de la embarcación). Lo relevante aquí es que es la primera vez que un actor no tradicional del sistema internacional, y ante este Tribunal, presenta un escrito de esa naturaleza en un proceso contencioso internacional. Esta acción pone de presente que las solicitudes de las organizaciones internacionales han de ser escuchadas a pesar del rechazo de algunos Estados. Así fue la posición rusa al considerar que no existe base legal para la concesión de la petición debido a la naturaleza no gubernamental de la organización que presentaba este escrito. Sin embargo, desde la perspectiva de este texto, es necesario reconocer el aporte que pueden generar estas organizaciones en el proceso coordinado de creación y fortalecimiento de instituciones internacionales, ya que son estas las que también pueden canalizar las demandas de los individuos en el sistema internacional. 


\section{Consideraciones finales}

En cuanto a su propia definición, si bien el concepto de gobernanza global va a tener una fuerte relación con la gobernanza a nivel doméstico, no tienen el mismo significado. Cuando nos referimos al escenario internacional, se hace referencia a Estados, que al menos en la forma gozan de la misma capacidad de decisión en el sistema, pese a que en la práctica poseen diferentes capacidades materiales que les dan una posición jerarquizada dentro de la comunidad internacional. Para el estudio del concepto de gobernanza global se proponen los siguientes atributos: dimensiones, rol del Estado, impacto sobre la soberanía, participación de actores no tradicionales, instituciones intergubernamentales, obstáculos en su desarrollo.

En cuanto a los actores que constituyen la gobernanza global, en este texto no se niega el Estado como un actor primordial del sistema, y por ello uno de los roles más importantes lo van a tener las organizaciones intergubernamentales. Se parte de la idea de que la comunidad internacional se encuentra lejos de ser una sociedad democrática, especialmente cuando se refiere a la influencia de otros actores; no obstante, no se puede desconocer el impacto que generan las ONG, ya que desarrollan un papel primordial a la hora de identificar los problemas que debe revisar la comunidad internacional e incluso en muchos de los procesos de tomas de decisiones. De esta manera, llegan a ser avaladas como un actor del sistema internacional, actores que con el tiempo han conseguido un asiento importante en la estructura de la gobernanza global.

En cuanto a las instituciones internacionales, si bien se logra la existencia de importantes acuerdos internacionales para gestionar los asuntos marítimos y una administración coordinada de estos, el verdadero desafío de las instituciones internacionales es generar un verdadero espacio de gestión orientada hacia la gobernanza global, el cual no necesariamente necesita de una renovación o modificación de estas, sino de un reconocimiento y observancia efectiva por parte de los Estados. Sin embargo, esto no implica que se desconozca la soberanía como un principio de las relaciones entre Estados. La invitación es a superar la discusión dualismo-monismo en cuanto a la relación del derecho doméstico y el derecho internacional y apostarle a un sistema coordinado de acoplamiento entre los sistemas y normas.

Respecto a la gestión de aguas internacionales, al parecer el verdadero problema en cuanto a gestión marítima son aquellas zonas donde el ejercicio de la soberanía no corresponde a un solo Estado, ya que en realidad lo que viene ocurriendo es que los Gobiernos más poderosos son los que se benefician de estos espacios. La alta mar parece ser un espacio sin ningún tipo de control, pero las consecuencias ambientales afectan de manera global sin ninguna especie de discriminación. Por ello, al ser más bien un juego de poderes y dominio, resulta el tema más difícil a la hora 
de hablar de gobernanza global; empero, organizaciones de diferente naturaleza han logrado generar suficiente presión para que sea un objeto de responsabilidad global compartida.

\section{Referencias}

Banco Mundial. (2017). World Development Report 2017: Governance and the Law. Washington, D. C.: autor.

Couvreur, P. (2007). La obra de la Corte Internacional de Justicia. Recuperado de http://www.ehu.eus/cursosderechointernacionalvitoria/ponencias/ pdf/2007/2007_4.pdf

David, C. P. (2008). La guerra y la paz. Enfoque contemporáneo sobre la seguridad y la estrategia. Barcelona: Icaria, Antrazyt.

Díez de Velasco, M. (2009). Instituciones de derecho internacional público (16ava. Ed.). Madrid: Tecnos.

Eberwein, W. D. (2010). Gobernanza internacional y Organizaciones no Gubernamentales. Revista del Centro de Estudios de Sociología del Trabajo (СЕSOT), (2), 2-32.

Gaviria Liévano, E. (2005). Derecho internacional público (6ta. Ed.). Bogotá: Temis.

Held, D. (1995). Democracy and the global order: From the modern state to cosmopolitan governance. Redwood City. Stanford University Press.

Held, D. (diciembre de 2010). Cosmopolitismo después del 11 de septiembre. Anales de la Cátedra Francisco Suárez, 44, 329-339.

Kaul, I. y Blondin, D. (2015). Los bienes públicos globales y las Naciones Unidas. En J. A. Ocampo (Ed.), Gobernanza global y desarrollo: nuevos desafíos $y$ prioridades de la cooperación internacional (pp. 71-114). Buenos Aires: Naciones Unidas, Siglo XxI.

Mayntz, R. (2001). El Estado y la sociedad civil en la gobernanza moderna. Reforma y Democracia, (21), 7-22.

Mayntz, R. (2002). Los Estados nacionales y la gobernanza global. Revista del CLAD. Reforma y Democracia, 24, 29-44.

Núñez Lozano, M. C. (2010). Hacia una política marítima integrada de la Unión Europea: estudios de política marítima. Madrid: Iustel. 
Organización de las Naciones Unidas (ONU). (1985). Convención de las Naciones Unidas sobre el derecho del mar: documento A/Conf. 62/122. En Tercera Conferencia de las Naciones Unidas sobre el Derecho del Mar.

Organización de las Naciones Unidas (ONU). (2004). Convención de las Naciones Unidas sobre el Derecho del Mar. Dirección de Intereses Marítimos. Recuperado de http://www.un.org/depts/los/convention_agreements/ texts/unclos/convemar_es.pdf

Paolillo, F. H. (1982). El impacto del nuevo derecho del mar en la evolución de la organización internacional. Estudios Internacionales, 15(59), 337-357.

Real Academia Española. (2014). Diccionario de la Lengua Española (23ava. Ed.). Madrid: autor.

Treves, T. (1998). Conflicts between the International Tribunal for the Law of the Sea and the International Court of Justice. New York University Journal of International Law and Politics, 31, 809-821.

Vargas C, E. (1975). La solución a las controversias en el derecho del mar. Revista Chilena de Derecho, 2(3-8), 207-222.

Vergara Molano, A. (1989). Derecho internacional público. Bogotá: Imprenta Nacional.

Wolf-Dieter, E. (2010). Gobernanza Internacional y Organizaciones no Gubernamentales. Revista del Centro de Estudios Sociológicos del Trabajo, (2), 2-32. 
